\title{
The inhibitory effect of CTAB on human osteosarcoma through the PI3K/AKT signaling pathway
}

\author{
WACILI DA, LIN TAO and YUE ZHU \\ Department of Orthopedics, The First Hospital of China Medical University, Shenyang, Liaoning 110001, P.R. China
}

Received February 27, 2021; Accepted April 16, 2021

DOI: $10.3892 /$ ijo.2021.5222

\begin{abstract}
Osteosarcoma (OS) metastasis and recurrence and multidrug resistance are three major obstacles in the clinic. New highly effective and low toxicity drugs for osteosarcoma are needed. The antitumoral efficacy of cetrimonium bromide (CTAB), a quaternary ammonium compound, is gradually being investigated. The aim of the present study was to investigate the effects of CTAB on OS cells and the underlying mechanisms. CTAB inhibited the proliferation of osteosarcoma cells in a concentration- and time-dependent manner, resulting in cell cycle arrest in G1 phase. CTAB also suppressed the migration and invasion of HOS and MG63 cells at a low concentration without inhibiting the growth of human osteoblasts. Moreover, CTAB promoted caspase-mediated apoptosis of osteosarcoma cells through the PI3K/AKT cascade, and this effect was accompanied by obvious mitochondrial toxicity. In vivo, CTAB inhibited OS proliferation without inducing organ toxicity. In conclusion, this study reveals that CTAB has an inhibitory effect on OS by suppressing proliferation and metastasis and inducing apoptosis through the PI3K/AKT signaling pathway and identifies CTAB as a potential therapeutic drug.
\end{abstract}

\section{Introduction}

Osteosarcoma (OS), one of the most common primary bone malignant tumors, usually occurs in children and adolescents aged 10-20 years; it is associated with a high degree of morbidity, and patients are prone to recurrence and metastasis, leading to a poor prognosis (1-3). Osteosarcoma tends to occur in areas where bone growth and bone turnover are more active, such as a typical long-axis medullary bone tumor in growing adolescents. Despite the innovative development

Correspondence to: Dr Lin Tao or Dr Yue Zhu, Department of Orthopedics, The First Hospital of China Medical University, 155 Nanjing North Street, Shenyang, Liaoning 110001, P.R. China

E-mail: taolindr@163.com

E-mail: zhuyuedr@163.com

Key words: osteosarcoma, CTAB, apoptosis, proliferation, migration, invasion, PI3K/AKT of neoadjuvant chemotherapy and surgery, which have the tremendous ability to shrink tumors and eliminate small lesions to ensure complete surgical resection and reduce tumor recurrence and metastasis, the 5-year survival rate of patients is less than $70 \%(4,5)$. This outcome may be related to various issues, such as complex pathogenesis, tumor heterogeneity, lack of novel adjuvant drugs, and an imperfect evaluation system. In addition, multidrug resistance caused by cross-resistance of chemotherapeutic drugs is a widely recognized problem (6). Previous findings have shown that alterations in the combination of chemotherapeutic drugs and the methods of administration do not improve 5-year survival, even when the doses are increased $(7,8)$. The major limitations of current clinical treatment regimens for osteosarcoma are recurrence and primary or secondary chemical resistance (9). Therefore, since surgical strategies have been developed, drug discovery is the key to improving survival rates.

In a previous study, a cell-based and phenotype-based high-throughput screening of approximately 2400 bioactive or clinically used compounds from the FDA-approved drug library (Selleck Chem) was conducted and it was found that, cetrimonium bromide (CTAB) has a tremendous inhibitory effect on osteosarcoma. CTAB, a quaternary ammonium compound, is used as a topical antiseptic and may play a variety of roles in cancer treatment, exhibiting the ability to penetrate the hydrophobic barriers of the plasma and mitochondrial membranes and accumulate in mitochondria under a negative transmembrane potential, which leads to mitochondrial toxicity $(10,11)$. Furthermore, the cation part of CTAB is the cause of bacterial cell wall damage, leading to the leakage of essential cell components. Notably, no adverse effects are observed in humans when CTAB is used as surgical lavage fluid, which also lays the foundation for our exploration of its effect on osteosarcoma $(12,13)$.

In summary, due to the urgency of exploring new drugs for osteosarcoma and the results of aforementioned studies, in the present study the effects of CTAB on the proliferation, apoptosis, invasion and metastasis of osteosarcoma and its underlying mechanisms were examined for the first time.

\section{Materials and methods}

Cells and animals. The human osteosarcoma cells (HOS, MG63 and U2OS) and human osteoblast line (hFOB1.19) used in this study were obtained from the Cell Bank of the 
Type Culture Collection of the Chinese Academy of Sciences (Shanghai, China). All of the cell lines were authenticated by Short Tandem Repeat (STR) profiling. The simian virus 40-transfected hFOB1.19 cells were applied to detect whether CTAB is toxic to normal human osteoblasts. The cells were cultured in high-glucose Dulbecco's modified Eagle's medium (DMEM; HyClone) supplemented with $10 \%$ fetal bovine serum (FBS; Invitrogen; Thermo Fisher Scientific, Inc.), and $1 \%$ penicillin/streptomycin (Sigma-Aldrich; Merck KGaA), and incubated at $37^{\circ} \mathrm{C}$ with $5 \% \mathrm{CO}_{2}$. BALB/c nude mice (male, 4-5 weeks, 20-22 g) were obtained from the Shanghai SLAC Laboratory Animal Co and housed in a standard animal environment $\left(22-26^{\circ} \mathrm{C}, 40-70 \%\right.$ humidity) with free access to water and food. All animal experiments were approved by the Animal Research Committee of the First Affiliated Hospital of Chinese Medical University.

Antibodies and reagents. CTAB was purchased from Sigma-Aldrich; Merck KGaA. PI3K agonist 740 Y-P were purchased from MedChemExpress (MCE). Antibodies against caspase-3 (1:1,000 dilution; cat. no. 9662), cleaved caspase-3 (1:1,000 dilution; cat. no. 9661), caspase-8 (1:1,000 dilution; cat. no. 9746), cleaved caspase-8 (1:1,000 dilution; cat. no. 9496), caspase-9 (1:1,000 dilution; cat. no. 9502), cleaved caspase-9 (1:1,000 dilution; cat. no. 20750), PARP (1:1,000 dilution; cat. no. 9532), cleaved PARP (1:1,000 dilution; cat. no. 32563), AKT (1:1,000 dilution; cat. no. 9272) and p-AKT (1:1,000 dilution; cat. no. 9271) were purchased from Cell Signaling Technology. Antibodies against PI3K (1:1,000 dilution; cat. no. 180967), p-PI3K (1:1,000 dilution; cat. no. 278545), Bcl-2 (1:1,000 dilution; cat. no. 32124), $\operatorname{Bax}$ (1:1,000 dilution; cat. no. 32503), cytochrome $c(1: 5,000$ dilution; cat. no. 133504), and $\beta$-actin (1:1,000 dilution; cat. no. 8226) and secondary antibodies (1:5,000 dilution; cat. no. 96899 and 96879) were obtained from Abcam. Phosphate-buffered saline (PBS) was obtained from Gibco; Thermo Fisher Scientific, Inc. Radioimmunoprecipitation assay (RIPA) lysis buffer was purchased from Santa Cruz Biotechnology.

Cell proliferation assay. OS cells and osteoblasts were cultured $\left(1 \times 10^{4}\right.$ cells/well) in 96-well plates (Thermo Fisher Scientific, Inc.) and treated with CTAB at different concentrations $(0,1,2,3,4,5,6,7$, and $8 \mu \mathrm{M})$ for 24,48 and $72 \mathrm{~h}$ in vitro. After the specified incubation time, $10 \mu 1$ of CCK- 8 (Dojindo Molecular Technologies, Inc.) was added to the plate, the cells were incubated at $37^{\circ} \mathrm{C}$ for $1-4 \mathrm{~h}$, and the absorbance was measured at $450 \mathrm{~nm}$ using an ELISA microplate reader (Bio-Rad). Cells without CTAB treatment were used as negative controls.

Cell cycle analysis. After incubation with a concentration gradient of CTAB at $37^{\circ} \mathrm{C}$ for $48 \mathrm{~h}$, the cells were collected, washed twice with PBS, and then fixed overnight with $70 \%$ cold ethanol at $4^{\circ} \mathrm{C}$. After the cells had been washed with PBS again, they were resuspended in $0.5 \mathrm{ml}$ stain buffer containing $100 \mu \mathrm{g} / \mathrm{ml}$ RNase A and $50 \mu \mathrm{g} / \mathrm{ml}$ PI (Beyotime Biotech) in the dark at room temperature for $30 \mathrm{~min}$ and analyzed by a flow cytometer (Becton-Dickinson). Moreover, CytExpert (version 2.3) was used for subsequent data analysis.
Cell metastasis assay. OS cell migration and invasion were detected by the wound-healing and Transwell assays, respectively. The cells were seeded in 6-well plates at a density of $5 \times 10^{5}$ cells/well. After the cell monolayer was formed, a micropipette tip was used to create a wound. Then, the cells were washed with PBS, and the medium was replaced with serum-free high-glucose DMEM containing different concentrations $(0,1,2$ and $4 \mu \mathrm{M})$ of CTAB. Cells migrating to the wound area were photographed with an inverted microscope at $0,6,18$ and $24 \mathrm{~h}$ (the average wound size represented the relative migration of cells). A 24-well Transwell chamber (Corning Costar) coated with matrix gel (Sigma-Aldrich; Merck KGaA) was used for cell invasion analysis. HOS and MG63 cells $\left(5 \times 10^{4}\right.$ cells/well) were inoculated with medium containing different concentrations ( 0 and $4 \mathrm{uM})$ of CTAB (without FBS) in the upper part of the Transwell chamber, and the lower chamber was filled with complete medium containing $10 \%$ FBS. After $24 \mathrm{~h}$ of treatment, the cells in the upper chamber were removed, and the remaining cells that had invaded through the Matrigel matrix were fixed with $4 \%$ paraformaldehyde, stained with $0.1 \%$ crystal violet, and counted under an inverted fluorescence microscope (Nikon Eclipse Ti-S).

Apoptosis analysis by flow cytometry. The effect of CTAB on the apoptosis of OS cells was analyzed by using a membrane protein V-FITC apoptosis detection kit (BD Biosciences). HOS and MG63 cells were inoculated in 6-well plates for $24 \mathrm{~h}$ and then treated with CTAB at a concentration of $0,2,4$ or $6 \mu \mathrm{M}$ for $48 \mathrm{~h}$. The cells were washed twice with precooled PBS, resuspended in $100 \mu 11 \mathrm{X}$ buffer and then incubated with FITC-labeled Annexin V as well as PI for $15 \mathrm{~min}$ at room temperature in the dark. Finally, $400 \mu 11 \mathrm{X}$ binding buffer was added to the reaction system, and cell apoptosis was assessed by flow cytometry (Becton-Dickinson) and CytExpert (version 2.3).

Western blot analysis. After treatment of HOS and MG63 cells with different doses of CTAB $(0,2,4$ and $6 \mu \mathrm{M})$ for $48 \mathrm{~h}$, the cells were washed twice with PBS and lysed with RIPA buffer comprising protease/phosphatase inhibitors to extract total protein. Mitochondrial and cytosolic proteins were isolated according to the protocol provided by the mitochondrial extract kit (Thermo Fisher Scientific Inc.). A BCA protein assay kit (Beyotime) was used to determine the protein concentration. Then, 10 and 12\% SDS-PAGE gels were used to separate the proteins (30 $\mu \mathrm{g}$ per lane), which were then transferred onto polyvinylidene fluoride (PVDF) membranes. The membranes were blocked with $5 \%$ bovine serum albumin solution at room temperature for $2 \mathrm{~h}$ and incubated with all the primary antibodies overnight at $4^{\circ} \mathrm{C}$. Then, the membranes were incubated with secondary antibody for $2 \mathrm{~h}$ at room temperature. Subsequently, the results were visualized with an enhanced chemiluminescence (ECL) system (UVP Inc.) and a Protein Blotting Detection and Imaging Scanner (Bio-Rad). ImageJ (NIH) software was used for quantitative analysis.

Mitochondrial membrane potential (MMP) assay. The change in the mitochondrial membrane potential (MMP) of osteosarcoma cells after intervention with CTAB was assessed 
by the JC-1 Analysis Kit (Beyotime). HOS and MG63 cells were seeded in 6 -well plates $\left(5 \times 10^{5} /\right.$ well) overnight and then treated with different doses of CTAB for $24 \mathrm{~h}$. The next day, the supernatant was removed, and the cells were washed with PBS and 1X JC-1 buffer. Then, the cells were resuspended in $500 \mu \mathrm{l} \mathrm{JC}-1$ staining solution in an incubator for $20 \mathrm{~min}$ and analyzed by flow cytometry (Beckham).

Tumor xenografts and histopathology. HOS cells $\left(2 \times 10^{6}\right)$ suspended in $100 \mu \mathrm{l}$ PBS were subcutaneously inoculated into the dorsal area of 4-week-old male nude mice. Three days after inoculation, the mice were randomly divided into 3 groups $(\mathrm{n}=4)$. Then, CTAB (10 or $20 \mathrm{mg} / \mathrm{kg} / \mathrm{d})$ was injected intraperitoneally every 3 days into each mouse for a total of 20 days. The control group was treated with the same volume of physiological saline. During the administration period, the body weights and tumor sizes of the mice were monitored every 3 days. The mice were sacrificed by cervical dislocation after 20 days of CTAB treatment, and the tumors and major organs, including the liver, lung, kidney, spleen and heart, were collected from each group of mice and immersed in $4 \%$ formalin for immunohistochemical staining and hematoxylin and eosin (H\&E) staining. The following formula was used to calculate the tumor volume: Volume $=1 / 2$ (length $\mathrm{x}$ width ${ }^{2}$ ). The tissue samples were fixed with $10 \%$ formalin (for $24 \mathrm{~h}$ at room temperature), embedded in paraffin, and cut into $4 \mu \mathrm{m}$ sections. The sections were deparaffinized with xylene and then dehydrated with gradient ethanol solutions. Then, the slides were stained with hematoxylin for $10 \mathrm{~min}$ and eosin for $5 \mathrm{~min}$. Tissue damage was observed under a microscope (Nikon) after the slides were sealed with neutral resin.

Statistical analysis. Data are expressed as the mean \pm standard deviation (SD). GraphPad Prism 7.0 software was used for statistical analysis, and Student's t-test or one-way analysis of variance (ANOVA) followed with Tukey post-hoc test was used to analyze differences between groups. $\mathrm{P}<0.05$ was considered statistically significant. All the cell experiments were performed in triplicate.

\section{Results}

$C T A B$ inhibited the proliferation of and induced cell cycle arrest in osteosarcoma cells. To investigate the effect of CTAB on the proliferation of OS cells, we treated HOS, MG63 and U2OS cells with different concentrations of CTAB in vitro for 24,48 or $72 \mathrm{~h}$. The results showed that CTAB significantly inhibited the activity of osteosarcoma cells in a time- and concentration-dependent manner (Fig. 1A), and the IC50 values of HOS, MG63, and U2OS cells were 4.949, 3.500, and 4.212 $\mu \mathrm{M}$, respectively (Fig. 1A). Notably, CTAB treatment did not affect the viability of hFOB1.19 cells (Fig. 1A), indicating that CTAB at a concentration of 1-8 $\mu \mathrm{M}$ had no significant toxicity compared to normal human osteoblasts. Based on the above results, 2, 4 and $6 \mu \mathrm{M}$ were selected as the effective drug concentrations for subsequent analysis. To determine whether CTAB inhibits cell proliferation by inducing cell cycle arrest, we evaluated the cell cycle distribution of osteosarcoma cells using flow cytometry. The proportion of G1 phase cells increased from 30.29 to $53.34 \%$, 40.23 to $61.55 \%$ and 39.95 to $60.58 \%$ in HOS, MG63 and U2OS cells treated with CTAB $(2,4$ or $6 \mu \mathrm{M})$, respectively (Fig. 1B). These data suggest that CTAB has an inhibitory effect on OS cells and induces G1 phase arrest.

$C T A B$ inhibits the migration and invasion of osteosarcoma cells. Tumor metastasis is the most common factor affecting the survival of patients. Therefore, inhibiting tumor migration and invasion may be an effective strategy to prevent tumor metastasis. In this study, the wound-healing assay showed that CTAB treatment significantly reduced the wound closure rate of HOS and MG63 cells in a dose-dependent manner (Fig. 2A). The invasion of tumor cells was analyzed by the Transwell assay, in which Matrigel matrix effectively simulates the invasion microenvironment of tumor cells. The digestion and penetration of Matrigel matrix by tumor cells is a good reflection of invasion ability. Compared with the control, CTAB significantly reduced the number of invasive HOS and MG63 cells (Fig. 2B).

CTAB induced apoptosis of osteosarcoma cells. The inhibitory effect of CTAB on the growth of osteosarcoma cells resulting from apoptosis was verified by flow cytometry. The percentage of apoptotic OS cells increased significantly after treatment with different concentrations of CTAB compared with control treatment for $48 \mathrm{~h}$ (Fig. 3A). The expression level of downstream apoptotic proteins was further measured by western blot analysis, and as shown in Fig. 3B and C, CTAB-treated OS cells exhibited downregulated expression of the antiapoptotic protein PARP and Bcl-2 but upregulated expression of cytosolic cytochrome $c$ (cyto-c) and the proapoptotic protein Bax. In addition, CTAB significantly promoted the cleavage of PARP, caspase-3, caspase- 8 and caspase- 9 . These results suggest that CTAB triggers apoptosis in OS cell lines. Disturbance of the mitochondrial membrane potential (MMP, $\Delta \Psi_{\mathrm{m}}$ ) is an early sign of apoptosis that affects the permeability of mitochondrial membranes (14). An increased mitochondrial membrane permeability leads to the release of mitochondrial apoptotic factors, such as cyto-c, which is transferred from the mitochondria to the cytoplasm and activates a series of apoptotic enzymes (15). The results of flow cytometry (Fig. 3D) showed that after $24 \mathrm{~h}$ of treatment with $4 \mu \mathrm{M}$ CTAB, the percentage of HOS and MG63 cells with a normal MMP was significantly reduced. These results indicate that CTAB may promote OS apoptosis by interfering with the MMP and disrupting mitochondrial membrane permeability.

$C T A B$ promoted osteosarcoma apoptosis by inhibiting the $P I 3 K-A K T$ pathway. The PI3K/AKT signaling pathway is the main intracellular signaling pathway that regulates the proliferation, apoptosis and migration of tumor cells (16). Previous findings have reported that excessive activation of the PI3K/AKT pathway is closely related to the negative regulation of tumor cell apoptosis (17). Therefore, we further analyzed whether CTAB-induced OS cell apoptosis depends on the PI3K/AKT signaling pathway. As shown in Fig. 4A, the expression levels of p-PI3k and p-AKT in HOS and MG63 cells treated with different concentrations of CTAB decreased significantly. We further investigated whether the 

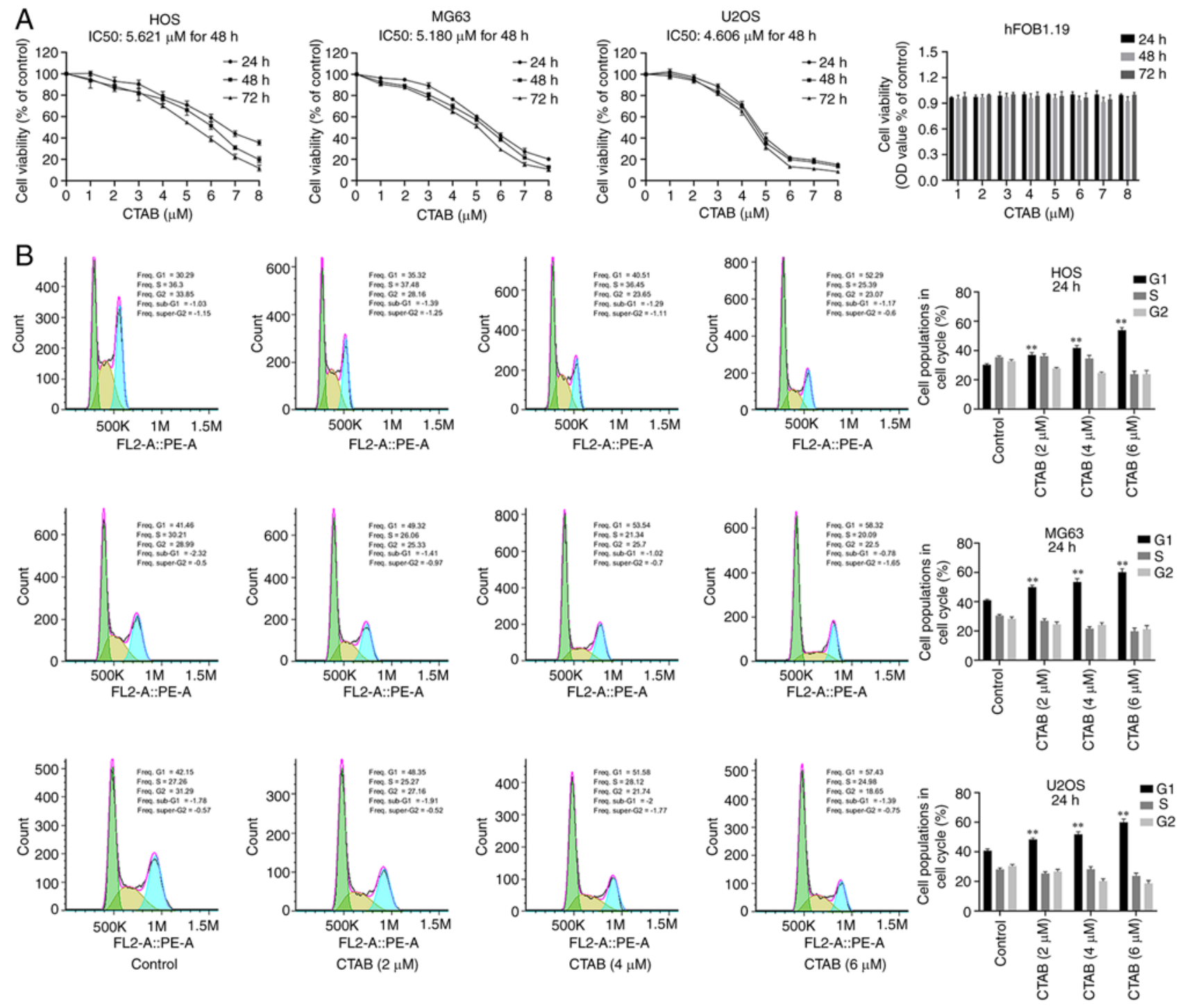

Figure 1. CTAB inhibits osteosarcoma cell proliferation and induces cell cycle arrest. (A) The CCK-8 assay was used to assess the proliferation of osteosarcoma cell lines (HOS, MG63 and U2OS) and a human osteoblast cell line (hFOB1.19) treated with different concentrations of CTAB for 24-72 h. (B) CTAB induced G1-phase arrest in HOS, MG63 and U2OS cells $\left(n=3,{ }^{* *} \mathrm{P}<0.01, \mathrm{CTAB}\right.$ compared with the control).

antitumor effect of CTAB is mediated through inhibition of the PI3K/AKT signaling pathway, and we found that 740Y-P (a PI3K agonist) partially reversed the inhibitory effect of CTAB on OS cells (Fig. 4B). Specifically, 740Y-P reversed CTAB-induced alterations in the abundance of p-PI3K, p-Akt, Bax and cleaved caspase-9 in HOS and MG63 cells (Fig. 4B). In addition, the flow cytometry showed that 740Y-P decreased the apoptosis rate of OS cells (Fig. 4C). After treatment with CTAB $(4 \mu \mathrm{M})$, the apoptosis rates of HOS and MG63 cells were 41.9 and $50.2 \%$, respectively, but the combination of CTAB and $740 Y-P$ reduced the apoptosis rate to 18.7 and $21.5 \%$, respectively. These results confirmed that CTAB induces apoptosis by inhibiting the PI3K/AKT signaling pathway.

$C T A B$ prevented the growth of OS cells in vivo. Since CTAB can inhibit OS cell proliferation and metastasis and induce apoptosis in vitro, we examined whether CTAB can inhibit the growth of OS (HOS) in xenograft tumors. As shown in Fig. 5A-D, CTAB treatment resulted in a significant reduction in tumor volume and weight in a dose-dependent manner. The average tumor volume of the control group was $1718 \pm 97 \mathrm{~mm}^{3}$, while that of the $10 \mathrm{mg} / \mathrm{kg} / \mathrm{d}$ CTAB-treated group was $987 \pm 107 \mathrm{~mm}^{3}$ and that of the $20 \mathrm{mg} / \mathrm{kg} / \mathrm{d}$ CTAB-treated group was $545 \pm 105 \mathrm{~mm}^{3}$. In addition, the mean tumor weight of the control group was $1380 \pm 87 \mathrm{mg}$, while that of the $10 \mathrm{mg} / \mathrm{kg} / \mathrm{d}$ CTAB-treated group was $566 \pm 101 \mathrm{mg}$ and that of the $20 \mathrm{mg} / \mathrm{kg} / \mathrm{d}$-treated group was $270 \pm 75 \mathrm{mg}$. In addition, there was no significant organ toxicity in the CTAB group, indicating that it safely exerts antitumor effects in vivo (Fig. 5E).

\section{Discussion}

With the rapid development of medical science and technology, the rate of limb salvage in osteosarcoma is greater than $80 \%$ in the clinic, and limb salvage has gradually replaced amputation in the majority of cases (18). Currently, the widely accepted strategy for osteosarcoma treatment is surgery combined with neoadjuvant chemotherapy $(19,20)$. Different chemotherapy regimens include the use of two to seven drugs, of which the four classic drugs that show 
A
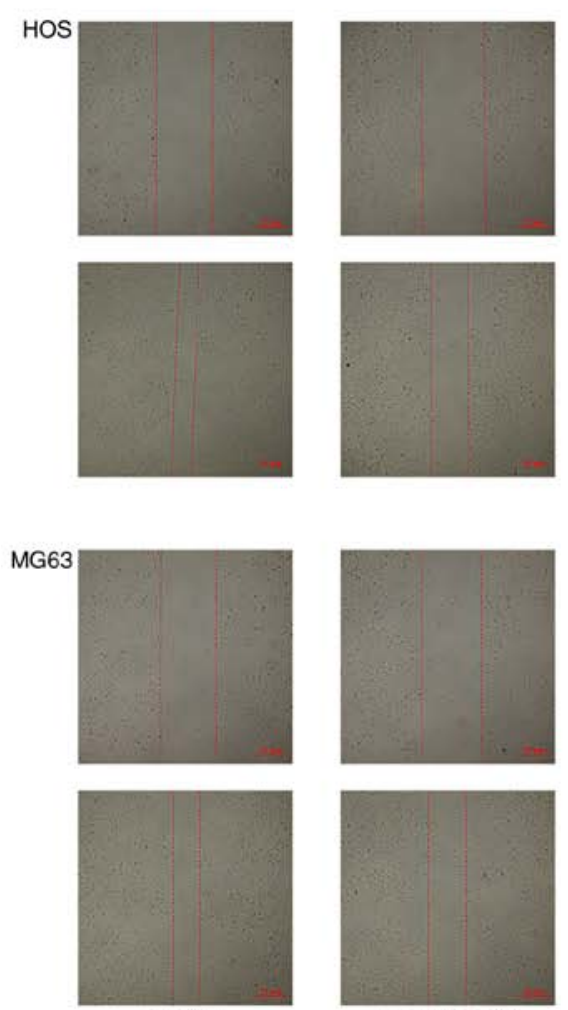

Control

B

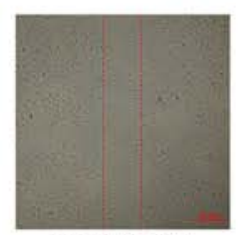

CTAB $(1 \mu \mathrm{M})$
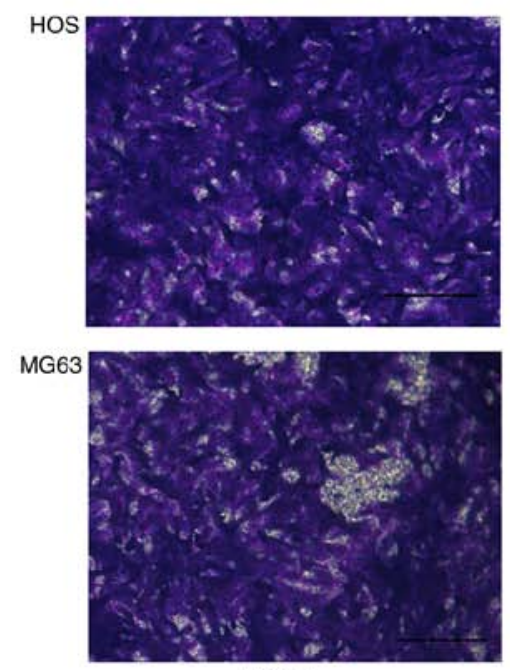

Control
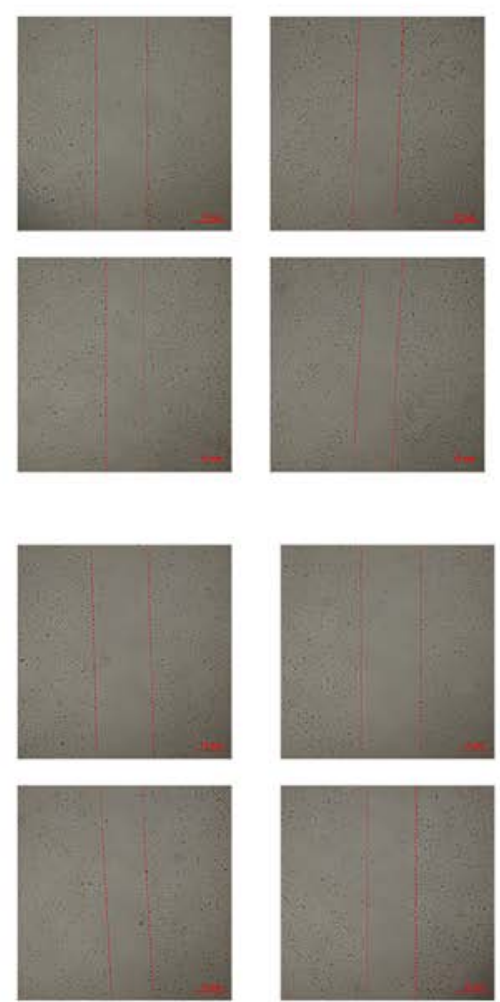

CTAB $(2 \mu \mathrm{M})$

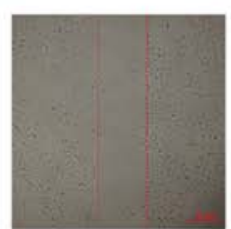

CTAB $(4 \mu \mathrm{M})$
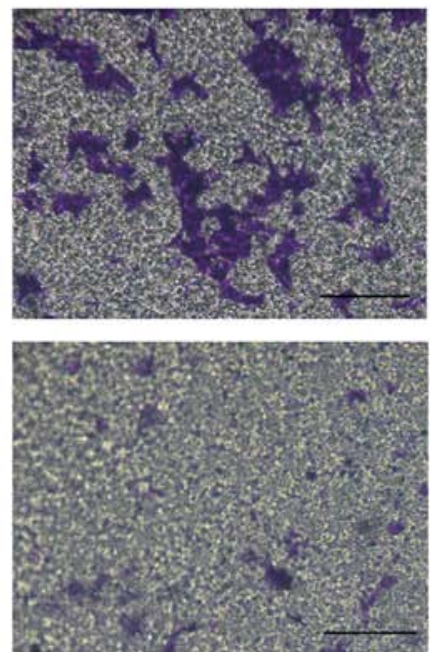

CTAB $(4 \mu \mathrm{M})$
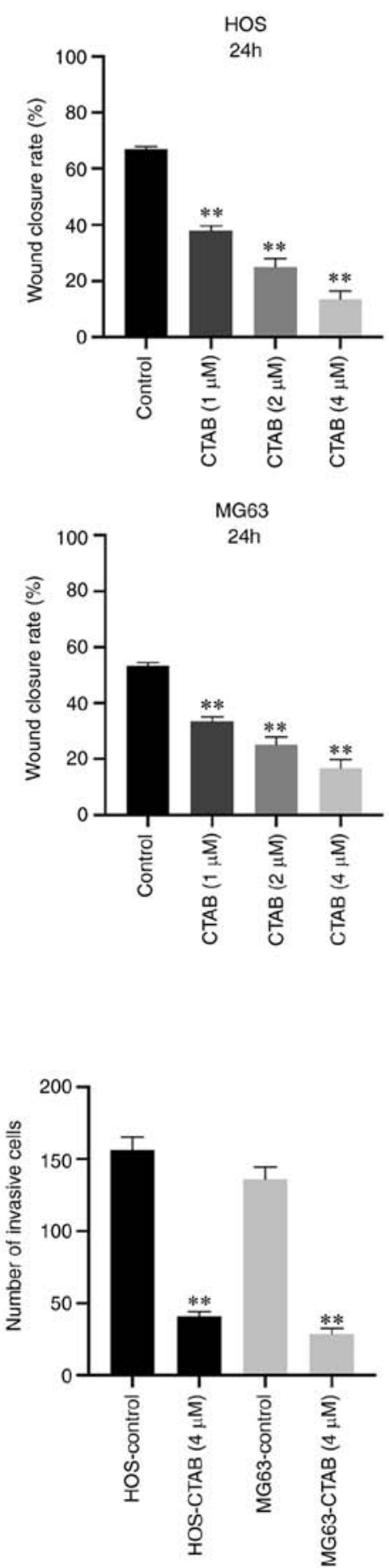

Figure 2. CTAB inhibits osteosarcoma cell migration and invasion. (A) The effects of CTAB on the migration of HOS and MG63 cells treated with CTAB for $24 \mathrm{~h}$ were determined by the wound-healing assay, and images were obtained with an inverted fluorescence microscope (magnification, $\mathrm{x} 40$; scale bar $=10 \mu \mathrm{M})$. (B) The effects of CTAB on the invasion of HOS and MG63 cells were assessed by a Matrigel Transwell assay (magnification, $\mathrm{x} 400$; scale bar=40 $\mu \mathrm{M}$ ) $\left(\mathrm{n}=3,{ }^{* *} \mathrm{P}<0.01, \mathrm{CTAB}\right.$ compared with the control).

consistent effects are cisplatin, doxorubicin, and high-dose methotrexate with leucovorin and ifosfamide with or without etoposide (21). With the development and application of neoadjuvant chemotherapies, the 5-year survival rate of patients with osteosarcoma has increased from $20 \%$ to more than $60 \%$. Moreover, another great value of neoadjuvant chemotherapy is that it provides time for patients to undergo artificial replacement without amputation. Therefore, preoperative chemotherapy-surgery-postoperative chemotherapy is the current standard treatment for osteosarcoma. However, attempts to target specific cell receptors and intracellular signaling molecules have not further improved survival rates due to the extreme genetic polymorphism of osteosarcoma cells. It is not difficult to determine that the key to improving the survival rate is drug therapy, which is also a challenging and controversial issue for orthopedic and oncology experts globally. We identified CTAB through high-throughput drug screening. CTAB is a known component of ctrimetide, 
$\mathrm{A}_{\mathrm{H}}$
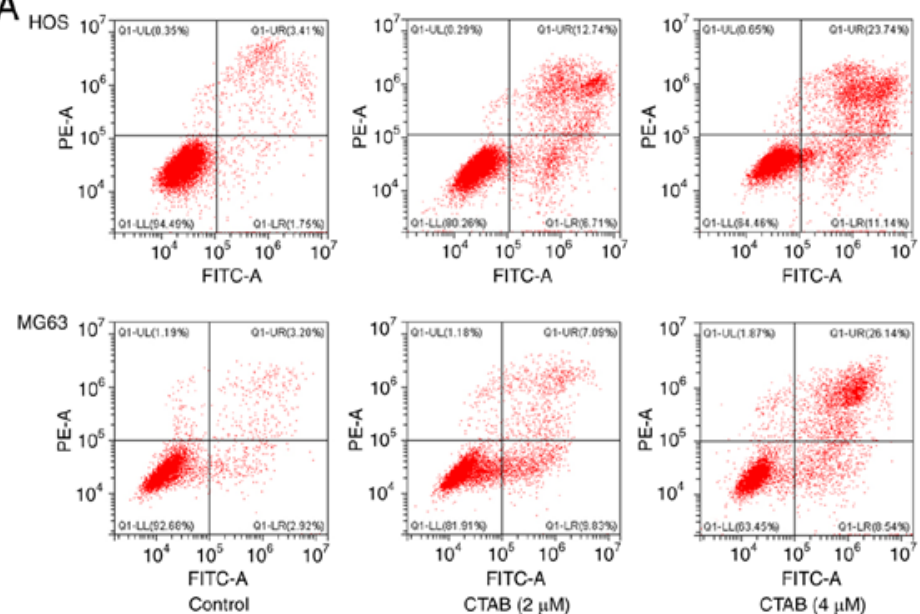

B

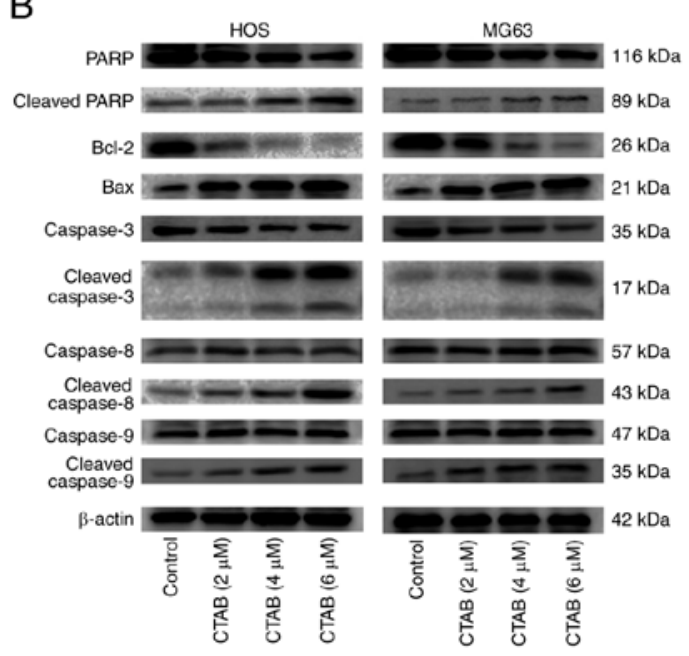

C

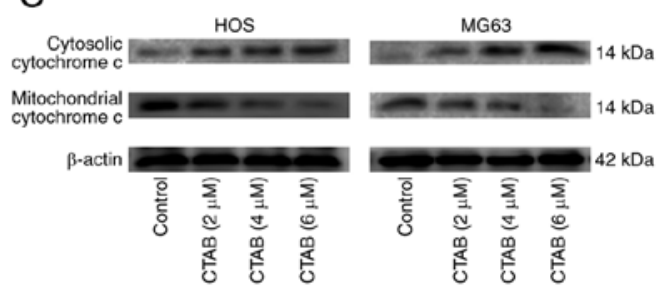

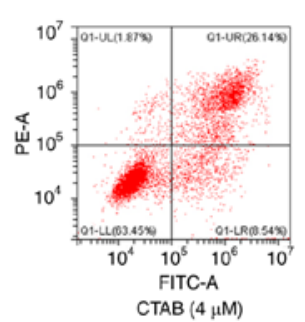

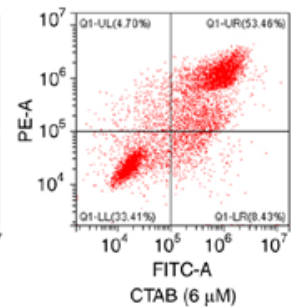

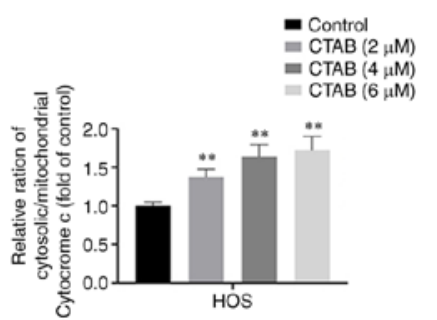

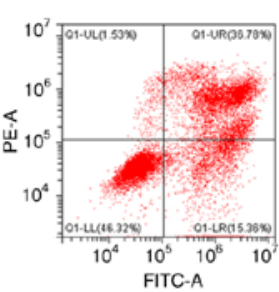

Hos
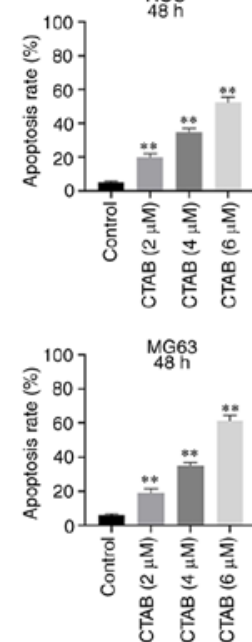

- Control

$=\mathrm{CTAB}(2 \mu \mathrm{M})$ $=\mathrm{CTAB}(4 \mu \mathrm{M})$
$=\mathrm{CTAB}(6 \mu \mathrm{M})$
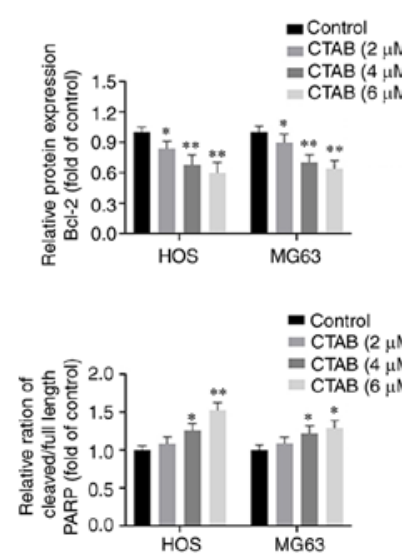

- Control $=$ CTAB $(2 \mu \mathrm{M}$
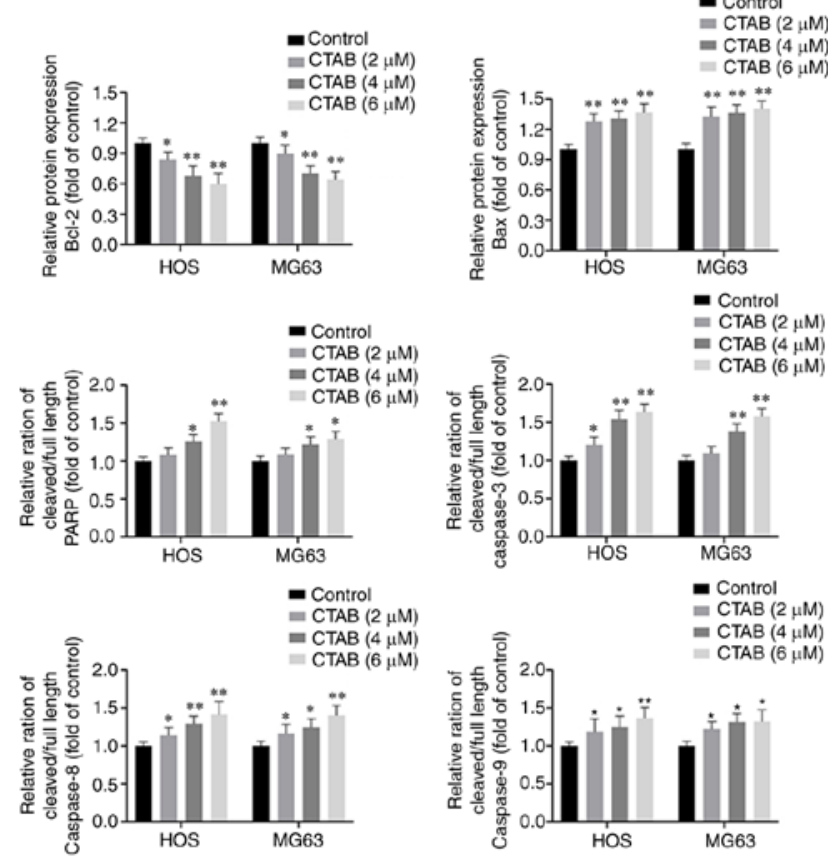

- Control - CTAB $(4 \mu \mathrm{M})$ $=$ CTAB $(4 \mu \mathrm{M}$
$=\operatorname{CTAB}(6 \mu \mathrm{M})$
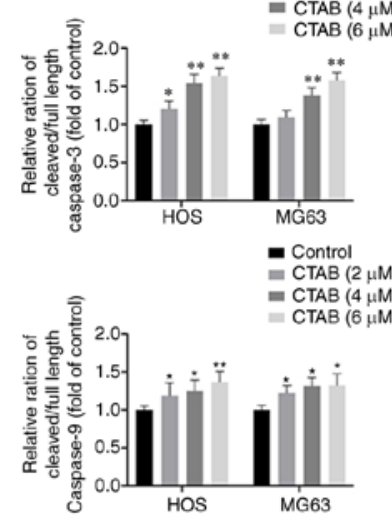

D
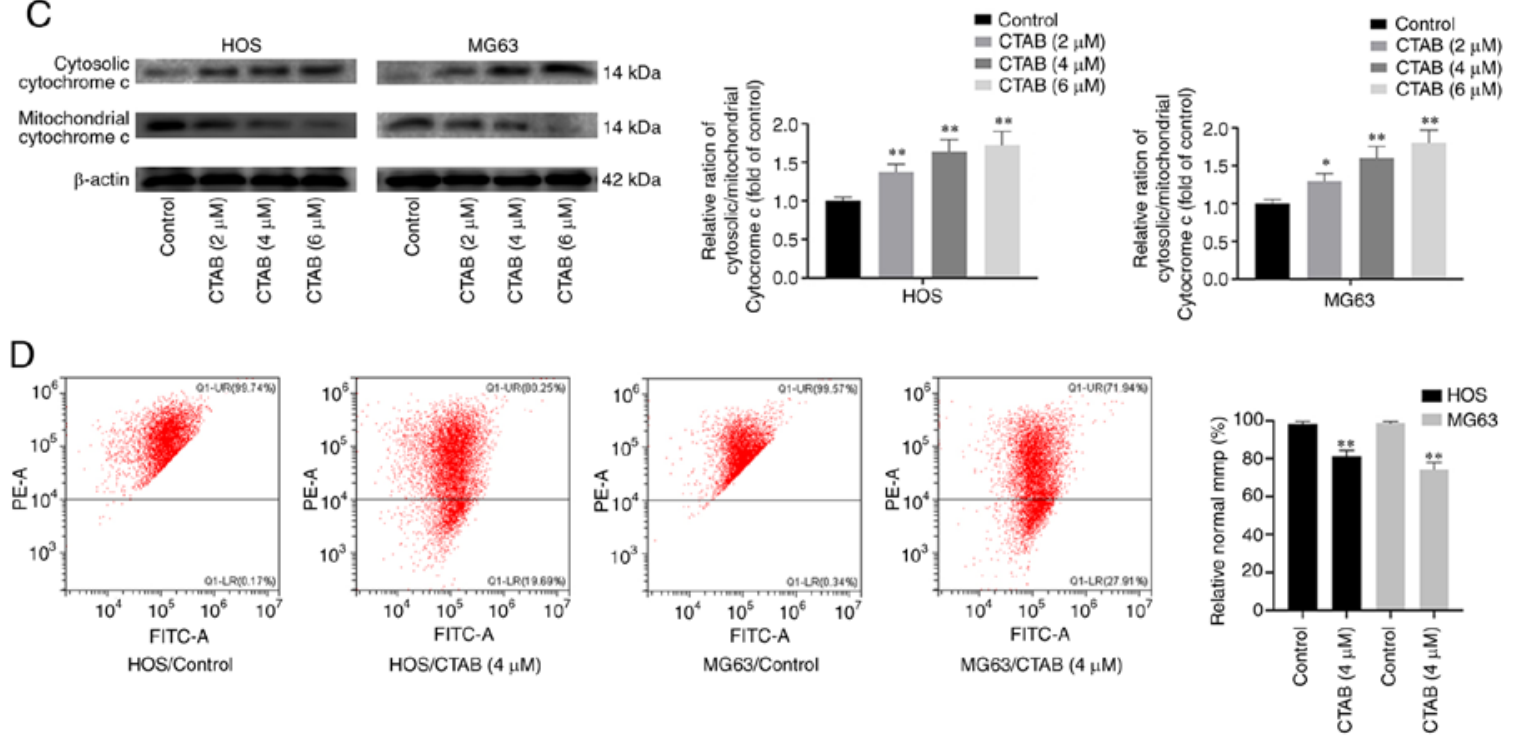

Figure 3. CTAB induces apoptosis of osteosarcoma cells. (A) Apoptosis of HOS and MG63 cells treated with different concentrations of CTAB (0,2,4 and $6 \mu \mathrm{M})$ for $48 \mathrm{~h}$ was determined by flow cytometry. (B) The expression of apoptosis-related proteins was measured by western blot analysis. (C) The expression of cytochrome $c$ (cyto-c) in cytoplasm and mitochondria was measured by western blot analysis. (D) The mitochondrial membrane potential (MMP) of HOS and MG63 cells treated with or without CTAB was determined by JC-1 using flow cytometry ( $\mathrm{n}=3$, ${ }^{*} \mathrm{P}<0.05$ and ${ }^{* *} \mathrm{P}<0.01, \mathrm{CTAB}$ compared with the control). 
A

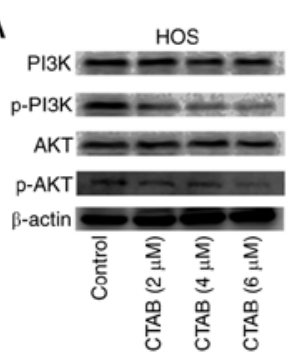

B

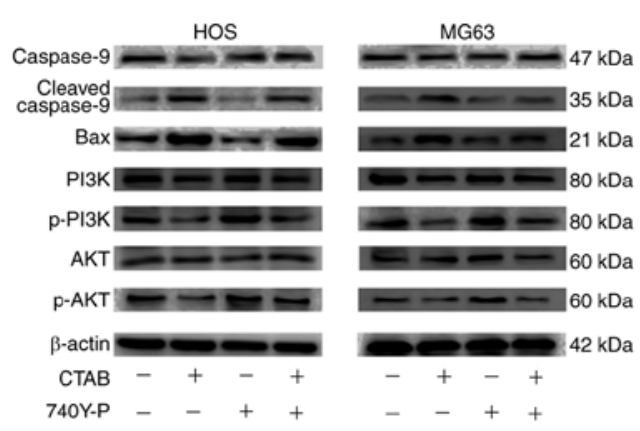
홍

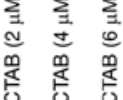

㠃
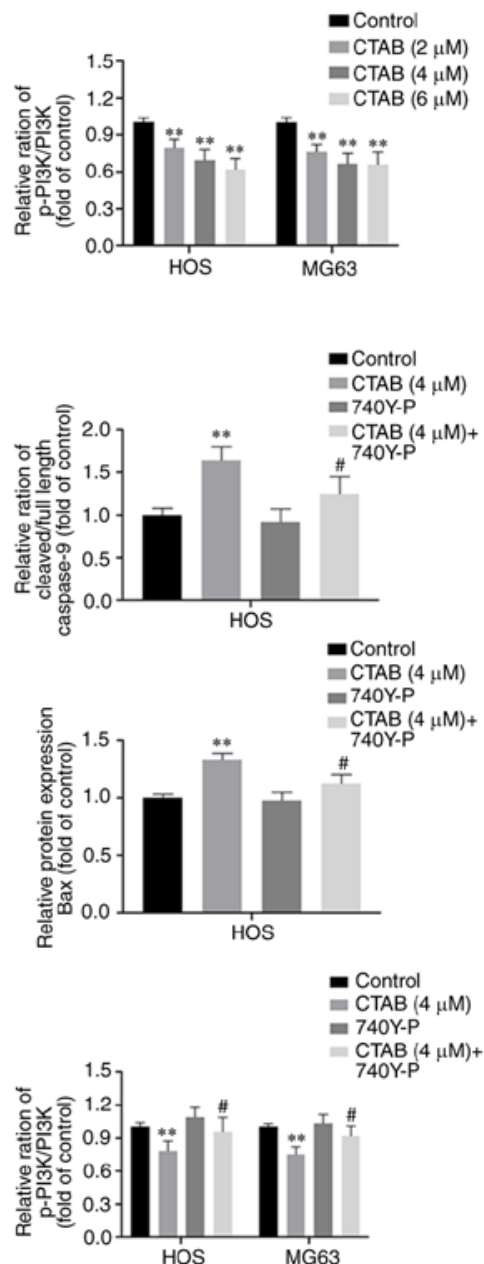
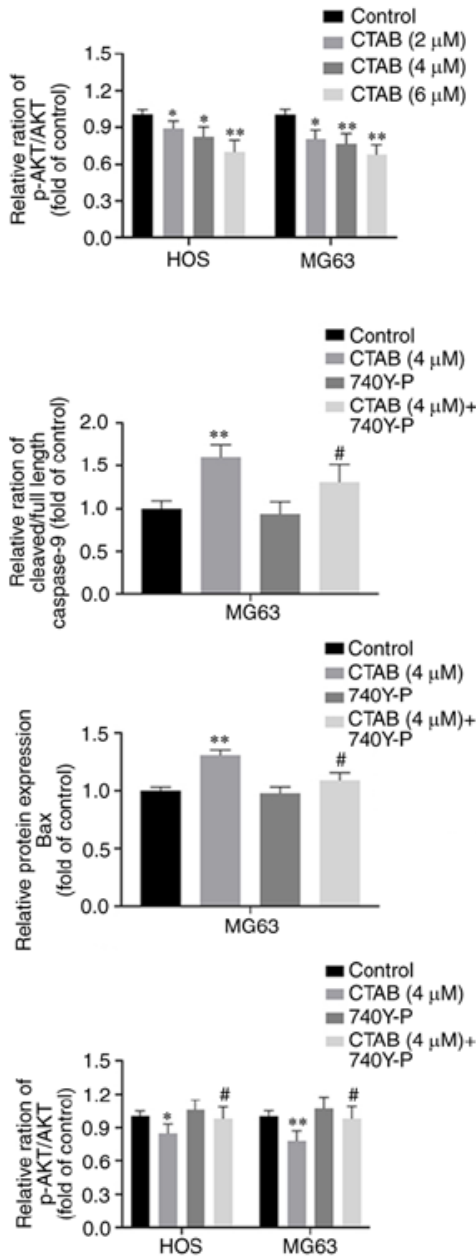

C
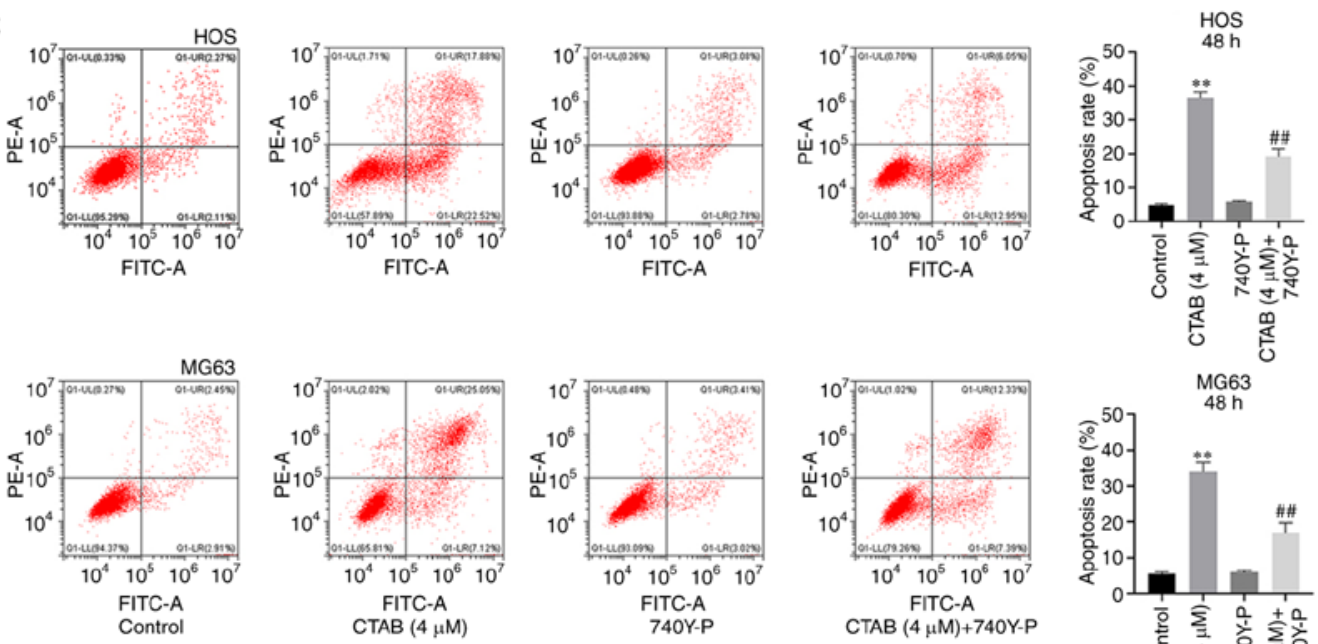

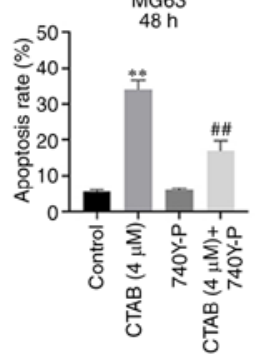

Figure 4. CTAB promotes osteosarcoma cell apoptosis by inhibiting the PI3K-AKT signaling pathway. (A) The expression of PI3K, p-PI3K, AKT and p-AKT in HOS and MG63 cells treated with different concentrations of CTAB for $48 \mathrm{~h}$ was analyzed by western blot analysis. (B) The expression of caspase-9, cleaved caspase-9, Bax, PI3K, p-PI3K, AKT and p-AKT in HOS and MG63 cells treated with CTAB $(4 \mu \mathrm{M})$ or CTAB $(4 \mu \mathrm{M})+740 \mathrm{Y}-\mathrm{P}(20 \mu \mathrm{M})$ for $48 \mathrm{~h}$ was measured by western blot analysis. (C) Apoptosis of HOS and MG63 cells was evaluated by flow cytometry ( $\mathrm{n}=3,{ }^{*} \mathrm{P}<0.05$ and ${ }^{* *} \mathrm{P}<0.01$, CTAB compared with the control; ${ }^{\#} \mathrm{P}<0.05$ and ${ }^{\# \prime} \mathrm{P}<0.01, \mathrm{CTAB}$ compared with $\left.\mathrm{CTAB}+740 \mathrm{Y}-\mathrm{P}\right)$.

which has been clinically used at clinically well-tolerated concentrations as a bactericidal adjuvant or tumor suppressor for hydatid cysts and during colorectal surgery $(12,13)$. However, no studies have explored the role of CTAB in osteosarcoma and its biochemical mechanisms. Our data suggest that CTAB significantly inhibits the proliferation of osteosarcoma cells, leading to G1 arrest, suppresses metastasis and induces apoptosis. 
A

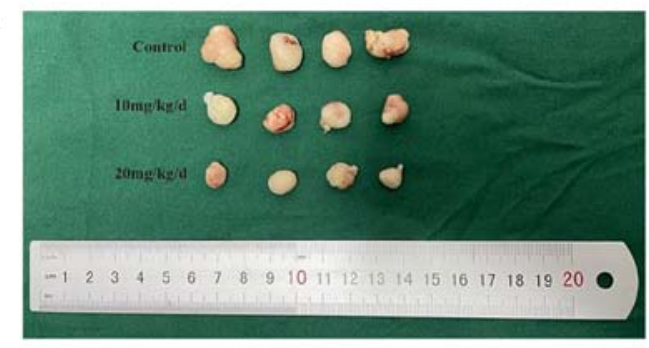

C

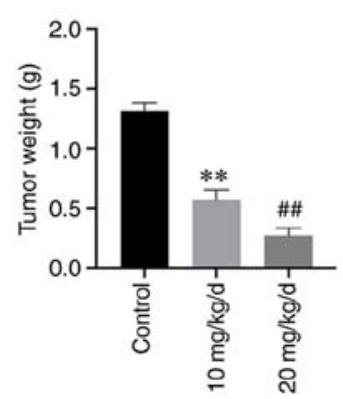

$E$
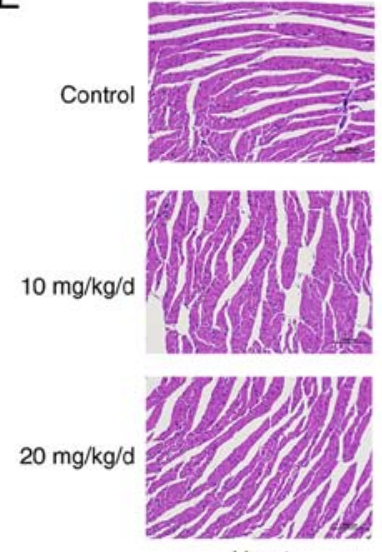

Heart
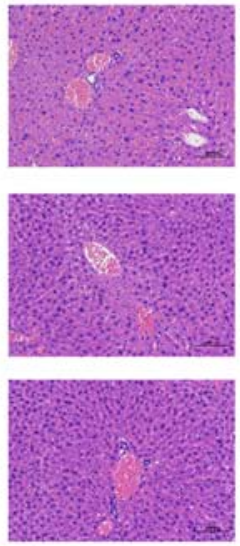

Liver
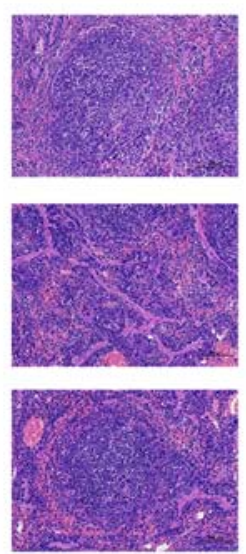

Spleen

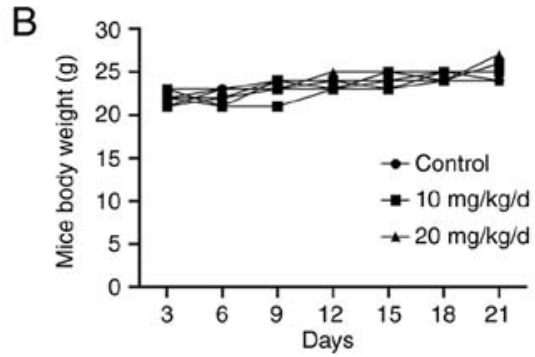

D

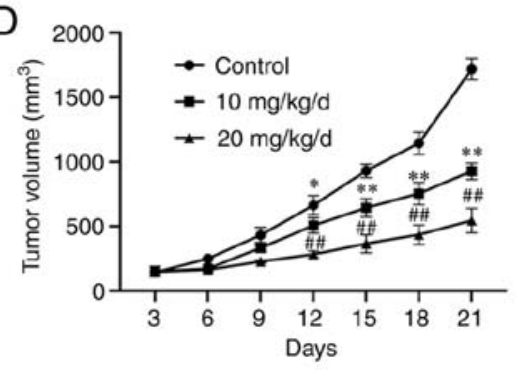

Figure 5. CTAB inhibits osteosarcoma cell (HOS) growth in mice. (A) Visual observation of osteosarcoma xenografts in BALB/C nude mice after 21 days of control or CTAB treatment. (B) Changes in the mass of mice over time. (C) Changes in the mass of tumors over time. (D) Changes in tumor size over time in mice. (E) H\&E staining was used to evaluate histologic changes in major organs $\left(\mathrm{n}=4,{ }^{*} \mathrm{P}<0.05\right.$ and ${ }^{* *} \mathrm{P}<0.01, \mathrm{CTAB}(10 \mathrm{mg} / \mathrm{kg} / \mathrm{d})$ compared with the control; ${ }^{\# \#} \mathrm{P}<0.01, \mathrm{CTAB}(20 \mathrm{mg} / \mathrm{kg} / \mathrm{d})$ compared with the control; magnification, $\mathrm{x} 200$; scale bar $\left.=100 \mu \mathrm{M}\right)$.

One of the clinical features of osteosarcoma is the tendency to form metastatic lesions, and the degradation of extracellular matrix is an essential step in cancer invasion and metastasis $(22,23)$. Previous findings have showed that CTAB can selectively inhibit the proliferation of prostate cancer cells and markedly decrease the invasion of DU-145 cells in the collagen matrix (24). In addition, CTAB has been used as an effective cytotoxic lavage solution in breast cancer surgery (25). CTAB may also suppress the invasion and metastasis of hepatocellular carcinoma cells by inhibiting TGF signal transduction (26). As bone metastasis is closely related to poor prognosis, controlling the invasion and metastasis of osteosarcoma cells is one of the difficulties in the treatment of osteosarcoma $(22,27)$. Osteosarcoma cells have the ability to migrate when cultured in vitro. The principle of the scratch test is the assessment of cell wound healing to detect the migration characteristics of cultured osteosarcoma cells. The Transwell experiment was performed to assess the invasion of osteosarcoma cells. We found that CTAB significantly inhibited the invasion and migration of HOS cells even at a low concentration. The ability of osteosarcoma cells to digest and penetrate Matrigel matrix after treatment with different concentrations of CTAB decreased significantly, and this was dependent on the concentration of drug.

Apoptosis is an energy-dependent, genetically programmed cell death mechanism that can be induced by either external (death receptors) or internal (mitochondria) pathways and is the main method for the elimination of tumors $(28,29)$. Various cancer therapies, such as chemotherapy, radiotherapy, immunotherapy and gene therapy, target the activation of apoptotic signal transduction pathways (30). Ito et al identified CTAB as a potential therapeutic agent for head and neck cancer due to its cytotoxic effects on related cell lines through mitochondria-mediated apoptosis pathways (31). In this study, CTAB intervention resulted in an increase in cleaved caspase-3, cleaved caspase- 8 and cleaved caspase- 9 expression in a dose-dependent manner, confirming that caspase-dependent apoptosis is involved in the cytotoxic effect of CTAB on osteosarcoma cells. The MMP was significantly reduced after CTAB treatment, indicating that CTAB can induce mitochondrial depolarization in OS cells. It is known that a reduction 
in the $\Delta \psi \mathrm{m}$ triggers the release of cytochrome $c$ from mitochondria into the cytoplasm, thereby initiating mitochondrial apoptosis signaling, and that the antiapoptotic Bcl-2 protein family participates in preventing the subsequent process (32). CTAB can increase the expression of proapoptotic Bax and downregulate the expression of antiapoptotic Bcl-2, thereby increasing mitochondrial permeability. The PI3K/AKT pathway is a widely studied intracellular signaling pathway that plays an indispensable role in all malignant phenotypes (such as tumorigenesis, cancer cell proliferation, survival, migration, and chemoresistance) $(33,34)$. According to reports, dysregulation of the PI3K/AKT pathway also plays a vital role in the occurrence and development of OS (35). Based on these previous studies, we found that CTAB intervention reduced the phosphorylation of PI3K/AKT in OS cells, indicating the inhibitory effect of CTAB on the PI3K/ AKT pathway. In addition, rescue experiments revealed that activation of the PI3K/AKT pathway by $740 \mathrm{Y}-\mathrm{P}$ abolished the inhibitory effect of CTAB on OS cell proliferation and apoptosis. These results indicated that CTAB inhibits cell activity and induces OS cell apoptosis by inhibiting the PI3K/AKT pathway. The key to exploring tumor cell radiotherapy and chemotherapy and antitumor drug screening is the establishment of tumor models. CTAB had no obvious toxic effects on and did not induce side effects on BALB/C-NU/nu nude mice, and no abnormalities in appetite, mental state or motor ability or obvious damage to the liver, spleen, kidney or other organs were observed. The aforementioned studies show that CTAB has a strong anti-osteosarcoma effect in nude mice without inducing obvious adverse drug reactions, which lays a good foundation for further research and clinical application. It is noteworthy that the antitumoral efficacy of CTAB has been studied for decades. Pan et al (36) also found the low toxicity of CTAB in vivo, which was consistent with the present study. Therefore, the focus of future research is exploring the drug delivery methods of CTAB in the treatment of osteosarcoma, such as local lavage, nanoparticle delivery system and gel sustained-release. Furthermore, future clinical studies need to be performed to assess whether administration of CTAB to osteosarcoma patients is beneficial.

In brief, osteosarcoma metastasis, recurrence and multidrug resistance (MDR) are the three major obstacles in the clinic. Since identification of anti-osteosarcoma drugs is imperative and because our study revealed the strong inhibitory effect of CTAB in osteosarcoma, we have sufficient reason to believe that CTAB is a potential therapeutic agent for osteosarcoma.

\section{Acknowledgements}

Not applicable.

\section{Funding}

No funding was received.

\section{Availability of data and materials}

The datasets supporting the conclusions of this article are available from the corresponding authors upon reasonable request.

\section{Authors' contributions}

WD, LT and YZ contributed to the study conception and design. Material preparation, data collection and analysis were performed by WD and LT. The first draft of the manuscript was written by WD. YZ was responsible for monitoring the progress of the entire study. YZ and LT confirmed the authenticity of the raw data. All authors read and approved the final manuscript.

\section{Ethics approval and consent to participate}

All animal experiments were approved by the Animal Research Committee of the First Affiliated Hospital of Chinese Medical University.

\section{Patient consent for publication}

Not applicable.

\section{Competing interests}

The authors declare that they have no competing interests.

\section{References}

1. Takeuchi A, Yamamoto N, Hayashi K, Matsubara H, Miwa S, Igarashi $\mathrm{K}$ and Tsuchiya $\mathrm{H}$ : Joint-preservation surgery for pediatric osteosarcoma of the knee joint. Cancer Metastasis Rev 38: 709-722, 2019.

2. Lilienthal I and Herold N: Targeting molecular mechanisms underlying treatment efficacy and resistance in osteosarcoma: A review of current and future strategies. Int J Mol Sci 21: 6885, 2020.

3. Hattinger CM, Patrizio MP, Magagnoli F, Luppi S and Serra M: An update on emerging drugs in osteosarcoma: Towards tailored therapies? Expert Opin Emerg Drugs 24: 153-171, 2019.

4. Ando K, Heymann MF, Stresing V, Mori K, Rédini F and Heymann D: Current therapeutic strategies and novel approaches in osteosarcoma. Cancers 5: 591-616, 2013.

5. Li B, Zhou P, Xu K, Chen T, Jiao J, Wei H, Yang X, Xu W, Wan W and Xiao J: Metformin induces cell cycle arrest, apoptosis and autophagy through ROS/JNK signaling pathway in human osteosarcoma. Int J Biol Sci 16: 74-84, 2020.

6. Ferrari S and Serra M: An update on chemotherapy for osteosarcoma. Expert Opin Pharmacother 16: 2727-2736, 2015.

7. Whelan JS, Jinks RC, McTiernan A, Sydes MR, Hook JM, Trani L, Uscinska B, Bramwell V, Lewis IJ, Nooij MA, et al: Survival from high-grade localised extremity osteosarcoma: Combined results and prognostic factors from three European Osteosarcoma Intergroup randomised controlled trials. Ann Oncol 23: 1607-1616, 2012.

8. Li S, Sun W, Wang H, Zuo D, Hua Y and Cai Z: Research progress on the multidrug resistance mechanisms of osteosarcoma chemotherapy and reversal. Tumour Biol 36: 1329-1338, 2015.

9. Anderson ME: Update on survival in osteosarcoma. Orthop Clin North Am 47: 283-292, 2016.

10. Chen LB: Mitochondrial membrane potential in living cells. Annu Rev Cell Biol 4: 155-181, 1988.

11. Pan Y, Wang Z, Shao D, Zheng H, Chen Y, Zheng X, Zhang M, $\mathrm{Li}$ J, Li F and Chen L: CTAB induced mitochondrial apoptosis by activating the AMPK-p53 pathway in hepatocarcinoma cells. Toxicol Res 4: 1359-1365,2015.

12. Umpleby HC and Williamson RC: The efficacy of agents employed to prevent anastomotic recurrence in colorectal carcinoma. Ann R Coll Surg Engl 66: 192-194, 1984.

13. Sonişik M, Korkmaz A, Besim H, Karayalçin K and Hamamci O: Efficacy of cetrimide-chlorhexidine combination in surgery for hydatid cyst. Br J Surg 85: 1277, 1998. 
14. Singh R, Letai A and Sarosiek K: Regulation of apoptosis in health and disease: The balancing act of BCL-2 family proteins. Nat Rev Mol Cell Biol 20: 175-193, 2019.

15. Kagan VE, Tyurin VA, Jiang J, Tyurina YY, Ritov VB, Amoscato AA, Osipov AN, Belikova NA, Kapralov AA, Kini V, et al: Cytochrome $\mathrm{c}$ acts as a cardiolipin oxygenase required for release of proapoptotic factors. Nat Chem Biol 1: 223-232, 2005.

16. Hennessy BT, Smith DL, Ram PT, Lu Y and Mills GB: Exploiting the PI3K/AKT pathway for cancer drug discovery. Nat Rev Drug Discov 4: 988-1004, 2005.

17. Delaloge S and DeForceville L: Targeting PI3K/AKT pathway in triple-negative breast cancer. Lancet Oncol 18: 1293-1294, 2017.

18. Jiang F, Shi Y, Li GJ and Zhou F: A meta-analysis of limb-salvage versus amputation in the treatment of patients with Enneking $\neq U$ pathologic fracture osteosarcoma. Indian J Cancer 51 (Suppl 2): e21-e24, 2015.

19. Yang C, Tian Y, Zhao F, Chen Z, Su P, Li Y and Qian A: Bone microenvironment and osteosarcoma metastasis. Int $\mathrm{J}$ Mol Sci 21: 6985, 2020.

20. Chindamo G, Sapino S, Peira E, Chirio D, Gonzalez MC and Gallarate M: Bone diseases: Current approach and future perspectives in drug delivery systems for bone targeted therapeutics. Nanomaterials (Basel) 10: 875, 2020.

21. Vos HI, Coenen MJ, Guchelaar HJ and Te Loo DM: The role of pharmacogenetics in the treatment of osteosarcoma. Drug Discov Today 21: 1775-1786, 2016.

22. Fu Y, Yu W, Cai $\mathrm{H}$ and $\mathrm{Lu} \mathrm{A}$ : Forecast of actin-binding proteins as the oncotarget in osteosarcoma-a review of mechanism, diagnosis and therapy. Onco Targets Ther 11: 1553-1561, 2018.

23. Cui J, Dean D, Hornicek FJ, Chen Z and Duan Z: The role of extracelluar matrix in osteosarcoma progression and metastasis. J Exp Clin Cancer Res 39: 178, 2020.

24. Wissing MD, Mendonca J, Kim E, Kim E, Shim JS, Kaelber NS, Kant H,Hammers H,Commes T, Van Diest PJ, et al: Identification of cetrimonium bromide and irinotecan as compounds with synthetic lethality against NDRG1 deficient prostate cancer cells. Cancer Biol Ther 14: 401-410, 2013.

25. Park K, Chetty U, Scott W and Miller W: The activity of locally applied cytotoxics to breast cancer cells in vitro. Ann R Coll Surg Engl 73: 96-99, 1991.
26. Wu TK, Chen $\mathrm{CH}$, Pan YR, Hu CW, Huang FM, Liu JY and Lee CJ: Cetrimonium bromide inhibits cell migration and invasion of human hepatic SK-HEP-1 cells through modulating the canonical and Non-canonical TGF- $\beta$ signaling pathways. Anticancer Res 39: 3621-3631, 2019.

27. Zhou J, Liu T and Wang W: Prognostic significance of matrix metalloproteinase 9 expression in osteosarcoma: A meta-analysis of 16 studies. Medicine (Baltimore) 97: e13051, 2018.

28. Wang G, Zhang T, Sun W, Wang H, Yin F, Wang Z, Zuo D, Sun M, Zhou Z, Lin B, et al: Arsenic sulfide induces apoptosis and autophagy through the activation of ROS/JNK and suppression of Akt/mTOR signaling pathways in osteosarcoma. Free Radic Biol Med 106: 24-37, 2017.

29. Bai L and Wang S: Targeting apoptosis pathways for new cancer therapeutics. Annu Rev Med 65: 139-155, 2014.

30. Mohamed MS, Bishr MK, Almutairi FM and Ali AG: Inhibitors of apoptosis: Clinical implications in cancer. Apoptosis 22: 1487-1509, 2017.

31. Ito E, Yip KW, Katz D, Fonseca SB, Hedley DW, Chow S, Xu GW Wood TE, Bastianutto C, Schimmer AD, et al: Potential use of cetrimonium bromide as an apoptosis-promoting anticancer agent for head and neck cancer. Mol Pharmacol 76: 969-983, 2009.

32. Bock FJ and Tait SWG: Mitochondria as multifaceted regulators of cell death. Nat Rev Mol Cell Biol 21: 85-100, 2020.

33. Liu R, Chen Y, Liu G, Li C, Song Y, Cao Z, Li W, Hu J, Lu C and Liu Y: PI3K/AKT pathway as a key link modulates the multidrug resistance of cancers. Cell Death Dis 11: 797, 2020.

34. Fattahi S, Amjadi-Moheb F, Tabaripour R, Ashrafi GH and Akhavan-Niaki H: PI3K/AKT/mTOR signaling in gastric cancer: Epigenetics and beyond. Life Sci 262: 118513, 2020.

35. Zhang H, Jiang H, Zhang H, Liu J, Hu X and Chen L: Anti-tumor efficacy of phellamurin in osteosarcoma cells: Involvement of the PI3K/AKT/mTOR pathway. Eur J Pharmacol 858: 172477, 2019.

36. Pan Y, Zhang Y, Chen Q, Tao X, Liu J and Xiao GG: CTAB enhances chemo-sensitivity through activation of AMPK signaling cascades in breast cancer. Front Pharmacol 10: 843, 2019.

(i) (9) This work is licensed under a Creative Commons Attribution-NonCommercial-NoDerivatives 4.0 International (CC BY-NC-ND 4.0) License. 